\title{
Response to Robert et al.: Terminal weaning or immediate extubation for withdrawing mechanical ventilation in critically ill patients (the ARREVE observational study)
}

\author{
Margaret L. Campbell \\ College of Nursing, Institution: Wayne State University, Address Line 1: 5557 Cass Ave., City: \\ Detroit, State: MI, Zip: 48202, Country: UNITED STATES
}

\section{Dear Editor}

I read with great interest the recent paper presenting the results of the ARREVE observational study comparing terminal extubation with terminal weaning. [1] The importance of this aspect of critical care research cannot be understated, given the number of patients who undergo ventilator withdrawal.

Patients undergoing ventilator withdrawal are a heterogenous group with regard to ability to experience respiratory distress during and following ventilator withdrawal. Some are awake and aware, most are cognitively impaired secondary to illness severity, and some are comatose. A singular approach to this procedure, extubation vs. weaning, as a unit custom or clinician preference does not take patient heterogeneity into consideration. An evidencebased algorithm guided by an objective measure of patient respiratory distress is needed to approach this procedure in a standardized manner that considers patient differences. My team conducted a pilot test of a ventilator withdrawal algorithm and found significantly more distress in the patients who underwent terminal extubation than in the patients whose withdrawal was algorithm guided.[2] We were not surprised to see similar results in the ARREVE study, with more distress displayed by the patients undergoing terminal extubation. We have a stepped-wedge cluster-randomized trial under way to establish in a powered sample the effectiveness of our algorithm (NCT03121391).

Although patient distress was a secondary outcome of the ARREVE study, we were surprised that a pain scale was used rather than a more sensitive measure of respiratory distress. We recommend the Respiratory Distress Observation Scale (RDOS) as both a clinical and a research tool. This eight-item scale has excellent psychometric properties, and cut-points for distress intensity have been established.[3, 4] In addition, the RDOS has been translated into Dutch, French, and Chinese. 


\section{References}

1. Robert R, et al. Terminal weaning or immediate extubation for withdrawing mechanical ventilation in critically ill patients (the ARREVE observational study). Intensive Care Med. 2017; 43:17931807. DOI: 10.1007/s00134-017-4891-0 [PubMed: 28936597]

2. Campbell ML, Yarandi HN, Mendez M. A two-group trial of a terminal ventilator withdrawal algorithm: pilot testing. J Palliat Med. 2015; 18(9):781-5. [PubMed: 26091245]

3. Campbell ML, Kero KK, Templin TN. Mild, moderate, and severe intensity cut-points for the Respiratory Distress Observation Scale. Heart Lung. 2017; 46(1):14-17. [PubMed: 27495878]

4. Campbell ML, Templin T, Walch J. A Respiratory Distress Observation Scale for patients unable to self-report dyspnea. J Palliat Med. 2010; 13(3):285-90. [PubMed: 20078243] 